\title{
Synthesis and Biological Properties of Luotonin A Derivatives
}

\author{
A. F. M. Motiur Rahman, Dong Hyeon Kim, Jing Lu Liang, Eung-Seok Lee, \\ Younghwa Na, Kyu-Yeon Jun, ${ }^{\ddagger}$ Youngjoo Kwon, ${ }^{\ddagger}$ and Yurngdong Jahng ${ }^{\star}$ \\ College of Pharmacy, Yetngnam University, Gyeongsan 712-749, Korea. "E-mail: ydiahng@umac.k. \\ ${ }^{\dagger}$ College of Phamacy, Catholic University of Daegu. Gyeongsan 712-702, Korea \\ "College of Pharmacy and Division of Life \& Pharmacettical Sciences, Ewha Womans Universin: Seoul 120-750. Korea \\ Received Atgust 1, 2008
}

\begin{abstract}
A series of new derivatives on the ring $A$ of luotonin $A$ were prepared by Friedlânder condensation of 6,7,8,10tetrahydropynolo[2,1-b]quinazoline-6,10-dione and suitably substituted 2-aminobenzaldehydes and 2aminoacetophenones. Their inhibitory activities on topoisonerases and cytotoxicities against selected human cancer cell lines were evaluated. Among the compounds tested, 8-fluoroluotonin A showed similar inhibitory activity on topoisomerase I comparable to camptothecin while luotonin A and 9-hydroxyluotonin A showed 1.37 and 0.94 times stronger inhibitory activity, respectively, on topoisonerase II compared to etoposide. Some derivatives of luotonin A showed moderate cytotoxicity. The possible relationship between the inhibitory activity on Topo II and the cytotoxicity of luotonin A and its analogues, thus, cannot be ruled out.
\end{abstract}

Key Words : Luotonin A, Topoisomerase I, Topoisomerase II, Cytotoxicity, Friedländer reaction

\section{Introduction}

Luotonin $\mathrm{A}$ is an alkaloid isolated from Peganmm nigellastrum Bunge (Zygophyllaceae) which has been used as a traditional oriental medical practice for the treatment of rheumatism, abscess, and inflammation. ${ }^{1}$ The basic fractions of $P$. nigellastrum showed anti-tumor activtity, ${ }^{2}$ and the origin of such an activity was recently revealed by identifying its constituent luotonin A which inhibited the growth of leukemia P-388 cells $\left(\mathrm{IC}_{50}=1.8 \mu \mathrm{g} / \mathrm{mL}\right)^{3}{ }^{3}$ Very recently, Hecht et $\mathrm{al}^{+}$have demonstrated that despite the lack of lactone ring functionality, luotonin A stabilizes the human DNA topoisomerase I (Topo I)-DNA covalent binary complex and mediates Topo I-dependent cytotoxicity in intact cells $\left(\mathrm{IC}_{5 i}=5.7-12.6 \mu \mathrm{m} / \mathrm{mL}\right)$, like camptothecin (CPT) and its analogues. ${ }^{4.5}$ Such intriguing properties of luotonin A not only led developments of efficient methods for total synthesis, ${ }^{6}$ but also introduced a variety of its derivatives. ${ }^{7}$ Studies on the derivatives of luotonin A were focused on the introduction of substituents on the ring $\mathrm{E}^{7}$ and adjustment of the ring size on the ring $\mathrm{C}^{8}$ To the best of our knowledge. only very limited numbers of compounds with (a) substituent(s) on the ring A and B were reported and shown promising biological properties. ${ }^{6 p, 7 \mathrm{~d}}$

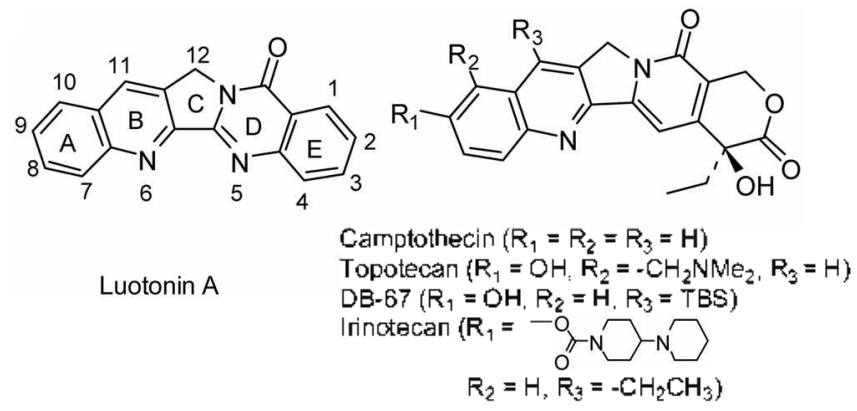

Although early crystal structure of the CPT-stabilized DNA-Topo I cleavage complex has revealed that the crucial $20-\mathrm{OH}$ of CPT is hydrogen bonded with Asp533 as well as ester moiety with Arg364 of Topo I, ${ }^{9}$ luotonin A, has shown similar interaction mode and shown promising cytotoxicity even though the functionalities in CPT are lacking. ${ }^{4.5}$

Our continuing interest in the search for biologically active agents derived from natural sources ${ }^{[i]}$ spurred us to design a series of luotonin A derivatives and to evaluate their biological properties.

\section{Results and Discussion}

Chemistry. Synthesis of the luotonin A derivatives 6 and 7 was straightforward as shown. Reactions of 1 with (substituted) 2-aminobenzaldehydes (2) were carried out under classical acidic Friedlander condition to afford the corresponding luotonin A derivatives (6) in fair yields. Hydrogenolysis of 9-benzyloxyluotonin A (6d) gave 9-hydroxyluotonin A (6h) in quantitative yield. Although most of the Friedländer condensation employed basic reaction condition, ${ }^{11}$ reactions of (substituted) 2-aminophenyl ketones (3) in the presence of $10-20 \% \mathrm{KOH}$ in $\mathrm{EtOH}$ were too sluggish to give the desired products. We, thus, pursued a step-wise reaction. The ketone 1a was treated with 2 -aminoacetophenone as well as substituted 2-aminoacetophenones in the presence of $p$-TsOH to afford corresponding Schiff's bases (5) in excellent yields (over 90\% in most cases). ${ }^{\mathrm{H}} \mathrm{H} \mathrm{XMR}$ spectra of 5, in fact, showed that more stable enamine-forms were the only forms. The resulting enamines were then subjected to Friedlander condition in the presence of Triton $\mathrm{B}$ to lead the desired compounds in good yields except $7 \mathbf{a}$. The prerequisite ketone $(1),{ }^{9}$ suitably substituted benzaldehydes $(2)^{12}$ and 2-aminoacetophenones $(3)^{13}$ were prepared by employing previously reported methods. 
In some cases, preparations of 2-aminoaldehydes are suffered from polymerization of resulting aminoaldehydes during reduction of 2-nitroaldehydes, which limits the scope of Friedlander reaction in the introduction of more substituents on Ring A.

$$
\text { (riton } \mathrm{B}
$$

It should be noted that Triton-B catalyzed cyclization of an intermediate enamine $5 \mathbf{a}$ resulted in $7 \mathbf{a}(14 \%)$ and unexpected compound 8 (41\%), of which the structure was confirmed by spectroscopic methods. The reaction mechanism for the formation of 8 remained to be explored.

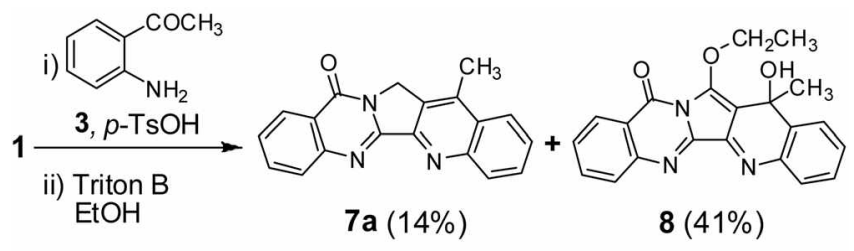

DNA topoisomerase inhibitory activity. Topo I and II inhibitory activities of the compounds prepared were measured by assessing the relaxation of supercoiled $\mathrm{pBR} 322$ plasmid DNA employing the method previously described. ${ }^{14}$ As shown in Table 1, 9-hydroxyluotonin A (6h) and 8fluoroluotonin A (6k) showed $54.1 \%$ and $81.1 \%$ inhibition at the concentration of $100 \mu \mathrm{M}$ which are comparable to $83.9 \%$ of CPT. In DNA Topo II assay, luotonin A (6a) and 6h are the most active inhibitors to show 73.5 and $52.4 \%$ inhibition comparable to $55.8 \%$ of etoposide at the concentration of $100 \mathrm{uM}$. It should be noted that the compounds (7a-d) with a methyl group at C11 did not show any promising inhibitory activity against Topo I and Topo II (data not shown). Although luotonin A has long been known as a Topo I inhibitor, present data implies that luotonin A can also be a good Topo II inhibitor.

Cytotoxicity. Cytotoxicities of compounds prepared were screened by the method previously reported ${ }^{15}$ against selected human cancer cell lines: HeLa (human cervix tumor), HCT-116 (human colon tumor), DU-145 (human prostate tumor), MDA-MB231 (human breast tumor), and HL-60 (human myeloid leukemic tumor) cell lines. As shown in Table $2, \mathrm{IC}_{50}$ values of compound prepared were ranged 19$86 \mu \mathrm{M}$ against HCT-116, DU-145, MDA-MB231, and HL60 while no activity was observed for HeLa up to $100 \mu \mathrm{M}$ (Table 2).

At the concentration of $100 \mu \mathrm{M}, 6 \mathrm{k}$ showed strong inhibitory activity on DNA Topo I compared with DNA Topo II with medium cytotoxicities against DU-145, MDAMB231, and HL-60. On the other hand, compounds 6a and

\begin{tabular}{|c|c|c|c|c|c|c|c|c|c|}
\hline & Reference & $6 a$ & $6 \mathrm{e}$ & $6 f$ & $6 \mathrm{~g}$ & $6 \mathrm{~h}$ & $6 \mathrm{j}$ & $6 \mathrm{k}$ & $6 n$ \\
\hline Topo I Inhibition & 83.9 & 33.7 & 16.7 & 11.2 & 12.9 & 54.1 & 17.4 & 81.1 & 19.2 \\
\hline Rel, potency & 1 & 0.40 & 0.20 & 0.13 & 0.15 & 0.65 & 0.21 & 0.97 & 0.23 \\
\hline Topo II Inhibition & 55.8 & 73.5 & 0 & 22.2 & 0 & 52.4 & 0 & 2.7 & 3.0 \\
\hline Rel, potency & 1 & 1.32 & - & 0.40 & - & 0.94 & - & 0.05 & 0.06 \\
\hline
\end{tabular}
6h showed stronger inhibitory activity against Topo II than against Topo I with relatively low, but selective cytotoxi-

Table 1. Inhibition of CPT. etoposide and selected luotonin A derivatives against Topo I and II

"Data were taken with 0.2 unit of Topo I and $100 \mu \mathrm{M}$ of CPT or compounds. "Data were takenl with 0.2 unit of Topo II and $100 \mu \mathrm{M}$ of etoposide or compounds

Table 2. Cytotoxic activity of luotonin $\mathrm{A}$ and its denvatives determined by cell proliferation assay

\begin{tabular}{cccccc}
\hline \multirow{2}{*}{ Compounds } & \multicolumn{5}{c}{ cell lines $\left(\mathrm{IC}_{\sin } / \mathrm{M}\right)^{\prime \prime}$} \\
\cline { 2 - 6 } & HeLa & HCT-116 & DU-145 & MDA-MB231 & HL-60 \\
\hline 6a & $33.83 \pm 3.45$ & $51.11=1.07$ & $23.25 \pm 5.95$ & $56.07 \pm 3.35$ & $>100$ \\
6h & $>100$ & $56.57 \pm 6.60$ & $>100$ & $47.41 \pm 0.25$ & $66.29=5.31$ \\
6k & $>100$ & $>100$ & $86.26 \pm 3.04$ & $49.95 \pm 0.02$ & $56.65=6.01$ \\
6n & $>100$ & $19.36 \pm 0.73$ & $27.83 \pm 2.24$ & $43.91 \pm 0.95$ & $21.78=2.49$ \\
Dauxorubicin & $7.46 \pm 0.41$ & $2.31 \pm 0.91$ & $4.78 \pm 0.21$ & $2.75 \pm 0.02$ & $0.72=0.08$ \\
Etoposide & $12.13 \pm 0.36$ & $14.94 \pm 1.59$ & $39.00 \pm 6.82$ & $22.15 \pm 0.32$ & $2.23=0.77$ \\
CPT & $7.84 \pm 0.27$ & $2.17 \pm 0.18$ & $5.51 \pm 1.14$ & $15.03 \pm 0.30$ & $0.06=0.02$ \\
\hline
\end{tabular}

“Each data point represents mean \pm S.D. from three different experiments performed in triplicate. Cell lines used are HeLa, human cervix tumor cell line: HCT 116, human colon tumor cell line: DU 145, human prostate tumor cell line: $\mathrm{VDA}-\mathrm{MB} 231$, liwman breast tumor cell line: HL-60. human myeloid leukemic tumor cell line. 
cities against HCT-116, MDA-MB231 and HL-60. It should be noted that $\mathbf{3 n}$ is not a good inhibitor of Topo I and II, but showed stronger cytotoxicities against HCT-116 and HL-60 with $\mathrm{IC}_{50}$ 's at the $20 \mu \mathrm{M}$ level. These results indicate that obvious correlation cannot be described between the cytotoxicity and the inhibitory activity of DNA relaxation and decatenation by DNA Topo I and II

In conclusion, a series of new derivatives of luotonin A on the ring $\mathrm{A}$ were prepared by Friedländer condensation of 6,7,8,10-tetrahydropyrrolo[2,1-b]quinazoline-6,10-dione and suitably substituted 2-aminobenzaldehydes and 2aminoacetophenones. Their inhibitory activities on topoisomerases and cytotoxicities against selected human cancer cell lines were evaluated to show that 8-fluoroluotonin A showed similar inhibitory activity on Topo I comparable to camptothecin while luotonin $\mathrm{A}$ and 9-hydroxyluotonin $\mathrm{A}$ 1.37 and 0.94 times stronger inhibition, respectively, on Topo II compared to etoposide. Some derivatives of luotonin A showed moderate cytotoxicity. The possible relationship between the inhibitory activity on Topo II and the cytotoxicity of luotonin A and its analogues, thus, cannot be ruled out.

\section{Experimental Section}

Melting points were determined using a Fischer-Jones melting points apparatus and are not corrected. IR spectra were taken from a Perkin-Elmer 1330 spectrophotometer. XMR spectra were obtained using a Bruker-250 spectrometer $250 \mathrm{MHz}$ or $300 \mathrm{MHz}$ for ${ }^{1} \mathrm{H}$ X.MR and $62.5 \mathrm{MHz}$ or $75 \mathrm{MHz}$ for ${ }^{13} \mathrm{C} \mathrm{NMR}$ and are reported as parts per million (ppm) from the internal standard tetramethylsilane (TMS). The starting materials $1 \mathrm{a},{ }^{8}{ }^{2},{ }^{12}$ and $\mathbf{3}^{13}$ were prepared by employing previously reported method. Chemicals and solvents were commercial reagent grade and used without further purification. Elemental analyses were taken on a Hewlett-Packard Model 185B elemental analyzer.

Luotonin A (6a) (General Procedure). A mixture of ketone (1) (48 $\mathrm{mg}, 0.24 \mathrm{mmol}$ ), 2-aminobenzaldehyde (29 $\mathrm{mg}, 1.2$ equiv.), and $p$-TsOH (20 mg) in toluene ( $20 \mathrm{~mL}$ ) was refluxed for $12 \mathrm{~h}$, cooled to room temperature, and diluted with hexane:ether (1:1). Resulting precipitate was collected and washed with hexane:ether $(1: 1)$ to afford $\mathbf{6 a}$ as a solid which was recrystallized from $\mathrm{EtOH}$ to give spectroscopically pure compound: $\mathrm{mp} 280-281^{\circ} \mathrm{C}$. The spectral data were identical to those of literature values.

9-Chloroluotonin A (6b): Pale yellow needles (78\%): mp: $233-234^{\circ} \mathrm{C} .{ }^{1} \mathrm{H} \mathrm{NMR}\left(\mathrm{CDCl}_{3}, 250 \mathrm{MHz}\right) \delta 8.42$ (d. $1 \mathrm{H}$. $J=8.3 \mathrm{~Hz}, \mathrm{H} 1), 8.38$ (d, $1 \mathrm{H}, J=8.3 \mathrm{~Hz}, \mathrm{H} 4), 8.36(\mathrm{~s}, 1 \mathrm{H}$, H11), 8.09 (d, 1H, $J=8.3 \mathrm{~Hz}, \mathrm{H} 7), 7.93(\mathrm{~d}, 1 \mathrm{H}, J=2.1 \mathrm{~Hz}$, $\mathrm{H} 10), 7.85(\mathrm{td}, 1 \mathrm{H}, J=8.3,1.0 \mathrm{~Hz}, \mathrm{H} 2), 7.77$ (dd, $1 \mathrm{H}, J=$ $9.0,2.1 \mathrm{~Hz}, \mathrm{H} 8), 7.58$ (td, $1 \mathrm{H}, J=8.0,1.0 \mathrm{~Hz}, \mathrm{H} 3), 5.34$ (s, $2 \mathrm{H}$ ). MS (ESI) Cacld. for $\mathrm{C}_{18} \mathrm{H}_{11} \mathrm{ClN}_{3} \mathrm{O}[\mathrm{M}+\mathrm{H}]^{-} 320$. Found 320. Anal. Calcd for $\mathrm{C}_{18} \mathrm{H}_{10} \mathrm{ClN}_{3} \mathrm{O} \mathrm{H}_{2} \mathrm{O}: \mathrm{C}, 64.01 ; \mathrm{H}, 3.58$; $\mathrm{x}, 12.44$. Found: $\mathrm{C}, 63.98 ; \mathrm{H}, 3.60 ; \mathrm{N}, 14.43$.

9-Fluoroluotonin A (6c): Pale yellow needles (78\%): mp: $273{ }^{\circ} \mathrm{C} .{ }^{1} \mathrm{H}$ NMR $\left(\mathrm{CDCl}_{3}, 250 \mathrm{MHz}\right) \delta 8.45\left(\mathrm{dd}, 1 \mathrm{H}, J_{\mathrm{H} \cdot \mathrm{F}}=\right.$ $\left.9.0 \mathrm{~Hz}, J_{\text {ortho }}=8.0 \mathrm{~Hz}, \mathrm{H} 10\right), 8.42(\mathrm{~d}, 1 \mathrm{H}, J=8.1 \mathrm{~Hz}, \mathrm{H} 1$ ), $8.39(\mathrm{~s}, 1 \mathrm{H}, \mathrm{H} 11), 8.18(\mathrm{~d}, 1 \mathrm{H}, J=8.1 \mathrm{~Hz}, \mathrm{H} 4), 7.84(\mathrm{td}, 1 \mathrm{H}$, $J=8.0,1.2 \mathrm{~Hz}, \mathrm{H} 2), 7.65-7.54(\mathrm{~m}, 3 \mathrm{H}), 5.33(\mathrm{~s}, 2 \mathrm{H}) . \mathrm{MS}$ (ESI) cacld. For $\mathrm{C}_{18} \mathrm{H}_{11} \mathrm{FN}_{3} \mathrm{O}[\mathrm{M}+\mathrm{H}]^{+}$304. Found 304. Anal. Calcd for $\mathrm{C}_{18} \mathrm{H}_{10} \mathrm{Fr}_{3} \mathrm{OH}_{2} \mathrm{O} ; \mathrm{C}, 67.29 ; \mathrm{H}, 3.76 ; \mathrm{N}, 13.08$. Found: $\mathrm{C}, 67.28 ; \mathrm{H}, 3.83 ; \mathrm{N}, 13.02$.

9-Bromoluotonin A (6d): Pale yellow needles (78\%): $\mathrm{mp}:>350{ }^{\circ} \mathrm{C} .{ }^{1} \mathrm{H} \mathrm{NMR}\left(\mathrm{CDCl} l_{3}, 250 \mathrm{MHz}\right) \delta 8.46(\mathrm{~s}, 1 \mathrm{H}$, H11), 8.42 (dd, $1 \mathrm{H}, J=8.3,0.8 \mathrm{~Hz}, \mathrm{H1}$ ), 8.18 (dd, $1 \mathrm{H}, J=$ $8.3,0.8 \mathrm{~Hz}, \mathrm{H} 4), 8.09(\mathrm{~d}, 1 \mathrm{H}, J=8.3 \mathrm{~Hz}, \mathrm{H} 7), 7.92(\mathrm{~d}, 1 \mathrm{H}, J$ $=7.5 \mathrm{~Hz}, \mathrm{H} 10), 7.85(\mathrm{td}, 1 \mathrm{H}, J=8.3,1.0 \mathrm{~Hz}, \mathrm{H} 3), 7.60-7.50$ $(\mathrm{m}, 2 \mathrm{H}), 5.36(\mathrm{~s}, 2 \mathrm{H})$. MS (ESI) Cacld. For $\mathrm{C}_{18} \mathrm{H}_{11} \mathrm{BrN}_{3} \mathrm{O}$ $[\mathrm{M}+\mathrm{H}]^{+}$364. Found 364. Anal. Calcd for $\mathrm{C}_{18} \mathrm{H}_{10} \mathrm{BrN}_{3} \mathrm{O}$ $0.5 \mathrm{H}_{2} \mathrm{O} ; \mathrm{C}, 57.93 ; \mathrm{H}, 2.97 ; \mathrm{X}, 11.26$. Found: $\mathrm{C}, 57.95 ; \mathrm{H}$, $2.99 ; \mathrm{N}, 11.26$.

9-Methoxyluotonin A (6e): Pale yellow needles (45\%): mp: $282{ }^{\circ} \mathrm{C} .{ }^{1} \mathrm{H} \mathrm{NMR}\left(\mathrm{CDCl}_{3}, 300 \mathrm{MHz}\right) \delta 8.38(\mathrm{dd}, 1 \mathrm{H}, J=$ $8.0,1.2 \mathrm{~Hz}, \mathrm{H} 1), 8.30(\mathrm{~d}, 1 \mathrm{H}, J=9.3 \mathrm{~Hz}, \mathrm{H} 4), 8.27(\mathrm{~s}, 1 \mathrm{H}$, $\mathrm{H} 14), 8.06(\mathrm{~d}, 1 \mathrm{H}, J=8.1 \mathrm{~Hz}, \mathrm{H} 7), 7.81$ (td, $1 \mathrm{H}, J=7.8,1.5$ $\mathrm{Hz}, \mathrm{H} 2) 7.53$ (td, $1 \mathrm{H}, J=7.6,1.0 \mathrm{~Hz}, \mathrm{H} 3$ ), 7.45 (dd, $1 \mathrm{H}, J=$ $9.3,2.7 \mathrm{~Hz}, \mathrm{H} 8), 7.11(\mathrm{~d}, 1 \mathrm{H}, J=2.7 \mathrm{~Hz}, \mathrm{H} 10), 5.62(\mathrm{~s}, 2 \mathrm{H})$, $3.94\left(\mathrm{~s}, 3 \mathrm{H},-\mathrm{OCH}_{3}\right) .{ }^{13} \mathrm{C}$ NMR $\left(\mathrm{CDCl}_{3}, 62.5 \mathrm{MHz}\right) \delta$ $160.70,159.35,152.77,149.43,148.59,145.69,134.52$, $132.04,130.31,130.02,129.72,128.58,127.16,126.40$, 123.97, 121.14, 104.99, 55.69 and 47.30. ESI MS Calcd for $\mathrm{C}_{1} \mathrm{H}_{1+}+\mathrm{N}_{3} \mathrm{O}_{2}[\mathrm{M}+\mathrm{H}]^{+}$316.3. Found 316.10 Anal. Calcd for $\mathrm{C}_{14} \mathrm{H}_{13} \mathrm{BrN}_{3} \mathrm{O}_{2}: \mathrm{C}, 72.37 ; \mathrm{H}, 4.16 ; \mathrm{N}, 13.33$. Found: $\mathrm{C}$, $72.39 ; \mathrm{H}, 4.15 ; \mathrm{N}, 13.32$.

9-Benzyloxyluotonin A (6f): Pale yellow needles (55\%): mp: $311{ }^{\circ} \mathrm{C} .{ }^{1} \mathrm{H} \times \mathrm{MR}\left(\mathrm{CDCl}_{3}, 250 \mathrm{MHz}\right) \delta 8.41$ (dd, $1 \mathrm{H}, J=$ $8.0,1.2 \mathrm{~Hz}, \mathrm{H} 1), 8.36(\mathrm{~d}, 1 \mathrm{H}, J=9.4 \mathrm{~Hz}, \mathrm{H} 4), 8.28(\mathrm{~s}, 1 \mathrm{H}$, $\mathrm{H} 11), 8.09(\mathrm{~d}, 1 \mathrm{H} . J=8.2 \mathrm{~Hz}, \mathrm{H} 7), 7.83(\mathrm{td}, 1 \mathrm{H}, J=7.7,1.3$ $\mathrm{Hz}, \mathrm{H} 2), 7.58-7.33(\mathrm{~m}, 8 \mathrm{H}), 5.29(\mathrm{~s}, 2 \mathrm{H}), 5.22(\mathrm{~s}, 2 \mathrm{H})$. MS (ESI) Calcd for $\mathrm{C}_{24} \mathrm{H}_{18} \mathrm{~N}_{3} \mathrm{O}_{2}[\mathrm{M}+\mathrm{H}]^{+}$. 392. Found 392. Anal. Calcd for $\mathrm{C}_{2} \mathrm{H}_{1} \mathrm{~N}_{3} \mathrm{O}_{2} \cdot 0.5 \mathrm{H}_{2} \mathrm{O}: \mathrm{C}, 74.99 ; \mathrm{H}, 4.53 ; \Upsilon, 10.49$. Found: $\mathrm{C}, 75.05 ; \mathrm{H}, 4.49 ; \mathrm{N}, 10.52$.

9-Acetyloxyluotonin A (6g): Pale yellow needles (47\%): mp: $212{ }^{\circ} \mathrm{C} .{ }^{1} \mathrm{H} \mathrm{NMR}\left(\mathrm{CD}_{3} \mathrm{OD}, 250 \mathrm{MHz}\right) \delta 8.45(\mathrm{~d}, 1 \mathrm{H}, J=$ $7.8 \mathrm{~Hz}, \mathrm{H1}), 8.39-8.37(\mathrm{~m}, 2 \mathrm{H}, \mathrm{H} 4$ and $\mathrm{H} 11), 8.07(\mathrm{~d}, 1 \mathrm{H}, J$ $=8.0 \mathrm{~Hz}, \mathrm{H} 7), 7.83(\mathrm{td}, 1 \mathrm{H}, J=8.0 .1 .0 \mathrm{~Hz}, \mathrm{H} 2), 7.78(\mathrm{~d}$, $1 \mathrm{H}, J=2.5 \mathrm{~Hz}, \mathrm{H} 10), 7.64(\mathrm{dd}, 1 \mathrm{H}, J=8.1,0.8 \mathrm{~Hz}, \mathrm{H} 8$ ), $7.56(\mathrm{t}, 1 \mathrm{H}, J=7.8 \mathrm{~Hz}, \mathrm{H3}), 5.32(\mathrm{~s}, 2 \mathrm{H}), 3.96(\mathrm{~s}, 3 \mathrm{H}) . \mathrm{MS}$ (ESI) Calcd for $\mathrm{C}_{20} \mathrm{H}_{14} \mathrm{~N}_{3} \mathrm{O}_{3}[\mathrm{M}+\mathrm{H}]^{+} 344$. Found $344 \mathrm{Anal}$. Calcd for $\mathrm{C}_{25} \mathrm{H}_{13} \mathrm{~N}_{3} \mathrm{O}_{3} \cdot 0.5 \mathrm{H}_{2} \mathrm{O}: \mathrm{C}, 68.18 ; \mathrm{H}, 4.00 ; \mathrm{N}, 11.93$. Found: $\mathrm{C}, 68.18 ; \mathrm{H}, 3.97 ; \mathrm{N}, 11.93$.

9-Hydroxyluotonin A (6h): A mixture of 9-benzyloxyluotonin $\mathrm{A}(39.1 \mathrm{mg}, 0.1 \mathrm{mmol})$ and $\mathrm{Pd} / \mathrm{C}(5 \%, 50 \mathrm{mg})$ in acetic acid $(5 \mathrm{~mL})$ was stirred under $\mathrm{H}_{2}$ atmosphere for 6 $\mathrm{h}$ and work-up as usual to give $30 \mathrm{mg}(99 \%)$ of white solid: $\mathrm{mp} 279^{\circ} \mathrm{C} .{ }^{\mathrm{l}} \mathrm{H} \mathrm{NMR}\left(\mathrm{CD}_{3} \mathrm{OD}, 250 \mathrm{MHz}\right) \delta 8.36(\mathrm{~s}, 1 \mathrm{H}$, H11), $8.30(\mathrm{~d}, 1 \mathrm{H}, J=8.0 \mathrm{~Hz}, \mathrm{H} 1), 8.10(\mathrm{~d}, 1 \mathrm{H}, J=8.0 \mathrm{~Hz}$, $\mathrm{H} 4), 7.93-7.84(\mathrm{~m}, 2 \mathrm{H}, \mathrm{H} 7$ and $\mathrm{H} 2), 7.57(\mathrm{td}, 1 \mathrm{H}, J=8.1,0.8$ $\mathrm{Hz}, \mathrm{H3}), 7.43(\mathrm{dd}, 1 \mathrm{H}, J=7.8,1.5 \mathrm{~Hz}, \mathrm{H} 8), 7.18(\mathrm{~d}, 1 \mathrm{H}, J=$ $1.0 \mathrm{~Hz}, \mathrm{H10}$ ), $5.28(\mathrm{~s}, 2 \mathrm{H})$. MS (ESI) Caled for $\mathrm{C}_{18} \mathrm{H}_{12} \mathrm{~N}_{3} \mathrm{O}_{2}$ $[\mathrm{M}+\mathrm{H}]^{+} 302$. Found 302 Anal. Calcd for $\mathrm{C}_{18} \mathrm{H}_{11} \mathrm{~N}_{3} \mathrm{O}_{2} \mathrm{H}_{2} \mathrm{O}$ : C, $67.71 ; \mathrm{H}, 4.10 ; \mathrm{N}, 13.16$. Found: C, 67.78; H, 4.13; $\mathrm{X}$, 13.13. 
8-Chloroluotonin A (6i): Pale yellow needles ( $36 \%$ ): $\mathrm{mp}$ : $286^{\circ} \mathrm{C} .{ }^{1} \mathrm{H} \times \mathrm{MR}\left(\mathrm{CDCl}_{5}, 250 \mathrm{MHz}\right) \delta 8.43(\mathrm{~s}, 2 \mathrm{H}, \mathrm{H} 7 \&$ H11), 8.42 (d, $1 \mathrm{H}, J=8.3 \mathrm{~Hz}, \mathrm{H1}), 8.11$ (d. $1 \mathrm{H}, J=8.3 \mathrm{~Hz}$, H4), 7.92-7.82(m, 2H), 7.65-7.55 (m, 2H), $5.32(\mathrm{~s}, 2 \mathrm{H})$. MS (ESI) Cacld for $\mathrm{C}_{18} \mathrm{H}_{11} \mathrm{CNN}_{3} \mathrm{O}[\mathrm{M}+\mathrm{H}]^{+} 320$. Found 320 . Anal. Calcd for $\mathrm{C}_{18} \mathrm{H}_{11} \mathrm{ClN} \mathrm{Cl}_{3} \mathrm{O} 0.5 \mathrm{H}_{2} \mathrm{O} ; \mathrm{C}, 65.76 ; \mathrm{H}, 3.37 ; \times$, 12.78. Found: C. $65.78 ; \mathrm{H}, 3.36 ; \mathrm{X}, 12.77$.

10-Chloroluotonin A (6j): Pale yellow needles $(78 \%)$; mp: $262{ }^{\circ} \mathrm{C} .{ }^{1} \mathrm{H} \times \mathrm{MR}\left(\mathrm{CDCl}_{3}, 250 \mathrm{MHz}\right) \delta 8.90(\mathrm{~s}, 1 \mathrm{H}$, H11), $8.43(\mathrm{~d}, 1 \mathrm{H}, J=8.3 \mathrm{~Hz}, \mathrm{H} 1), 8.40(\mathrm{~d}, 1 \mathrm{H}, J=8.3 \mathrm{~Hz}$. H4), 8.11 (d, $1 \mathrm{H}, J=8.3 \mathrm{~Hz}, \mathrm{H} 7), 7.86$ (t, $1 \mathrm{H}, J=7.5 \mathrm{~Hz}$. $\mathrm{H} 2), 7.78-7.72(\mathrm{~m}, 2 \mathrm{H}, \mathrm{H} 8$ and $\mathrm{H} 9), 7.59(\mathrm{td}, 1 \mathrm{H}, J=8.0,1.0$ $\mathrm{Hz}, \mathrm{H} 3) .5 .39$ (s, 2H). MS (ESI) Cacld for $\mathrm{C}_{18} \mathrm{H}_{11} \mathrm{ClN}_{3} \mathrm{O}$ $[\mathrm{M}+\mathrm{H}]^{+}$320. Found 320. Anal. Calcd for $\mathrm{C}_{18} \mathrm{H}_{10} \mathrm{ClN}_{3} \mathrm{O} \mathrm{H}_{2} \mathrm{O}$ : C. $64.01 ; \mathrm{H}, 3.58 ; \mathrm{X}, 12.44$. Found: C. $63.98 ; \mathrm{H}, 3.60 ; \mathrm{X}$. 14.43 .

8-Fluoroluotonin A (6k): Pale yellow needles (39\%): mp: $269^{\circ} \mathrm{C} .{ }^{1} \mathrm{H} \times \mathrm{MR}\left(\mathrm{CDCl}_{3}, 250 \mathrm{MHz}\right) \delta 8.45(\mathrm{~s}, 1 \mathrm{H}$, H11), 8.42 (dd. $1 \mathrm{H}, J=8.1,1.5 \mathrm{~Hz}, \mathrm{H1}), 8.12-8.05$ (m, $2 \mathrm{H}$. $\mathrm{H} 4$ and $\mathrm{H} 7$ ), 7.96 (dd, $\left.1 \mathrm{H} . J_{\mathrm{H}-\mathrm{F}}=9.0, J=8.0 \mathrm{~Hz}, \mathrm{H} 10\right), 7.86$ (td, $1 \mathrm{H} . J=8.0,1.0 \mathrm{~Hz}, \mathrm{H} 3), 7.58$ (td, $1 \mathrm{H} . J=8.0,1.0 \mathrm{~Hz}$. H3), 7.49 (ddd, $\left.1 \mathrm{H}, J_{\mathrm{H}-\mathrm{F}}=9.0, J=8.0,1.0 \mathrm{~Hz}, \mathrm{H} 9\right), 5.30$ (s. $2 \mathrm{H}$ ). MS (ESI) Cacld for $\mathrm{C}_{18} \mathrm{H}_{11} \mathrm{FN}_{5} \mathrm{O}[\mathrm{M}+\mathrm{H}]^{-}$304. Found 304. Anal. Calcd for $\mathrm{C}_{18} \mathrm{H}_{10} \mathrm{FN}_{3} \mathrm{O} \mathrm{H}_{2} \mathrm{O}: \mathrm{C}, 67.29 ; \mathrm{H}, 3.76 ; \mathrm{X}$, 13.08. Found: C. $67.32 ; \mathrm{H}, 3.69 ; \mathrm{X}, 13.12$.

8,9-Dimethoxyluotonin A (61): Pale yellow needles (49\%): mp $294{ }^{\circ} \mathrm{C} .{ }^{~} \mathrm{H} \times \mathrm{MR}\left(\mathrm{CDCl}_{3}, 250 \mathrm{MHz}\right) \delta 8.41(\mathrm{~d}$. $1 \mathrm{H}, J=7.5 \mathrm{~Hz}, \mathrm{H1}), 8.25(\mathrm{~s}, 1 \mathrm{H}, \mathrm{H} 11), 8.06(\mathrm{~d}, 1 \mathrm{H} . J=8.0$ $\mathrm{Hz}, \mathrm{H} 4), 7.82(\mathrm{t}, 1 \mathrm{H}, \mathrm{J}=8.0 \mathrm{~Hz}, \mathrm{H} 3), 7.75(\mathrm{~s}, 1 \mathrm{H}, \mathrm{H} 7), 7.58$ (td, $1 \mathrm{H}, J=7.8,1.3 \mathrm{~Hz}, \mathrm{H} 2), 7.13$ (s, 1H, H10), 5.27 (s, 2H), 4.05 (s, 6H). MS (ESI) Cacld. for $\mathrm{C}_{2 j} \mathrm{H}_{16} \mathrm{~N}_{3} \mathrm{O}_{3}[\mathrm{M}+\mathrm{H}]^{+} 346$. Found 346. Anal. Calcd for $\mathrm{C}_{2 j} \mathrm{H}_{1} \leqq \mathrm{~N}_{3} \mathrm{O}_{3} \mathrm{H}_{2} \mathrm{O}: \mathrm{C}, 66.11 ; \mathrm{H}$. $4.72 ; \mathrm{X}, 11.56$. Found: $\mathrm{C}, 66.17 ; \mathrm{H}, 4.69 ; \mathrm{N}, 11.53$.

8,9-Difluoroluotonin A $(6 \mathrm{~m})$ : Pale yellow needles $(43 \%): \mathrm{mp} 245^{\circ} \mathrm{C} .{ }^{1} \mathrm{H}$ NMR $\left(\mathrm{CDCl}_{3}, 250 \mathrm{MHz}\right) \delta 8.35(\mathrm{~s}$. $1 \mathrm{H}, \mathrm{H} 11), 8.33$ (d, $1 \mathrm{H}, J=7.5 \mathrm{~Hz}, \mathrm{H1}), 8.17$ (ddd, $1 \mathrm{H} . J_{\mathrm{H} \cdot \mathrm{F}}=$ $\left.10.0, J_{\mathrm{H}-\mathrm{F}}=8.0 \mathrm{~Hz}, J_{\text {para }}=1.0 \mathrm{~Hz}, \mathrm{H} 7\right), 8.06(\mathrm{~d}, 1 \mathrm{H} . J=8.0$ $\mathrm{Hz}, \mathrm{H} 4), 7.83(\mathrm{t}, 1 \mathrm{H}, J=8.0 \mathrm{~Hz}, \mathrm{H} 2), 7.67\left(\mathrm{dd}, 1 \mathrm{H} . J_{\mathrm{H}-\mathrm{F}}=\right.$ $\left.10.0 \mathrm{~Hz}, J_{\mathrm{H} \cdot \mathrm{F}}=8.0 \mathrm{~Hz}, \mathrm{H} 10\right), 7.56(\mathrm{t}, 1 \mathrm{H}, J=7.8 \mathrm{~Hz}, \mathrm{H} 3$ ), 5.30 (s, $2 \mathrm{H}$ ). MS (ESI) Cacld. for $\mathrm{C}_{18} \mathrm{H}_{10} \mathrm{~F}_{2} \mathrm{~N}_{3} \mathrm{O}[\mathrm{M}+\mathrm{H}]^{+}$ 322. Found 322. Anal. Calcd for $\mathrm{C}_{18} \mathrm{H}_{10} \mathrm{~F}_{2} \mathrm{~N}_{3} \mathrm{OH}_{2} \mathrm{O}$ : C, $63.72 ; \mathrm{H}, 3.27 ; \mathrm{N}, 11.20$. Found: C, 63.67; H, 2.29; N, 11.19.

8,9-Methylenedioxyluotonin A (6n): Pale yellow needles (88\%): mp: $336^{\circ} \mathrm{C} .{ }^{l} \mathrm{H} \times \mathrm{MR}\left(\mathrm{CDCl}_{3}, 250 \mathrm{MHz}\right) \delta 8.38(\mathrm{~d}$. $1 \mathrm{H}, J=8.0 \mathrm{~Hz}, \mathrm{H1}$ ), 8.18 (s, $1 \mathrm{H}, \mathrm{H} 11), 8.05$ (d, $1 \mathrm{H}, J=8.0$ $\mathrm{Hz}, \mathrm{H} 4), 7.81(\mathrm{td}, 1 \mathrm{H} . J=7.5,1.5 \mathrm{~Hz}, \mathrm{H} 2), 7.66(\mathrm{~s}, 1 \mathrm{H}, \mathrm{H} 7)$, $7.53(\mathrm{td}, 1 \mathrm{H}, J=7.7,1.3 \mathrm{~Hz}, \mathrm{H} 3), 7.09$ (s, $1 \mathrm{H}, \mathrm{H} 10), 6.16$ (s, $2 \mathrm{H}), 5.28(\mathrm{~s}, 2 \mathrm{H}) .{ }^{13} \mathrm{C} \mathrm{NMR}\left(\mathrm{CDCl}_{3}, 62.5 \mathrm{MHz}\right) \delta 160.58$. $152.79,151.79,149.62,149.33,148.55,148.07,134.46$. $129.85,128.58,128.46,127.04,126.73,126.31,121.02$, 106.35, 102.61, 102.36, 47.18. MS (ESI) Cacld. for $\mathrm{C}_{10} \mathrm{H}_{12} \mathrm{~N}_{3} \mathrm{O}_{3}$ $[\mathrm{M}+\mathrm{H}]^{+}$330. Found 330. Anal. Calcd for $\mathrm{C}_{20} \mathrm{H}_{11} \mathrm{~N}_{3} \mathrm{O}_{3} \cdot \mathrm{H}_{2} \mathrm{O}$ : C. $66.70 ; \mathrm{H}, 3.77 ; \mathrm{X}, 12.10$. Found: C. $66.67 ; \mathrm{H}, 3.79 ; \mathrm{X}$, 12.09 .

11-Methylluotonin A (7a): To a solution of ketone 1 (48 $\mathrm{mg}, 0.24 \mathrm{mmol}$ ) and 2-aminoacetophenone $(3,0.40 \mathrm{mg}, 1.2$ equiv.) in toluene $(20 \mathrm{~mL})$ was added $p$-TsOH $(10 \mathrm{mg})$.
Resulting mixture was refluxed stirred for $12 \mathrm{~h}$, cooled to room temperature, and diluted with hexane:ether (1:1). Resulting precipitate was collected and washed with hexane: ether (1:1) to afford the corresponding enamine (5) as a solid, which was recrystallized, from EtOH to give spectroscopically pure compound. Into the solution of the compound obtained in absolute $\mathrm{EtOH}(10 \mathrm{~mL})$ was added triton $\mathrm{B}(0.1 \mathrm{~mL})$ and resulting reaction mixture was refluxed for $12 \mathrm{~h}$. Cooling the reaction mixture resulted in precipitation which was collected and washed with cold $\mathrm{EtOH}$ to give yellow solid. The crude product was chromatographed on silica gel eluting with hexane:EtOAc (1:1). The early fractions $\left(R_{\mathrm{f}}=0.4\right)$ give $30 \mathrm{mg}(41 \%)$ of 14-hydroxy-14-methylquino[2,3:3,4]pyrrolo[2,1-b] quinazolin-13-ethyloxy-11-one (8) as a violet powder: $\mathrm{mp} 220-222{ }^{\circ} \mathrm{C} .{ }^{~} \mathrm{H}$ NMR $\left(\mathrm{CDCl}_{3}\right.$, $250 \mathrm{MHz}) \delta 8.35(\mathrm{~d}, 1 \mathrm{H}, J=8.0 \mathrm{~Hz}, \mathrm{H1}), 8.15(\mathrm{~d}, 1 \mathrm{H}, J=$ $8.3 \mathrm{~Hz}, \mathrm{H} 4), 8.10$ (dd, $1 \mathrm{H}, J=8.3,1.0 \mathrm{~Hz}, \mathrm{H} 7$ ), 7.83 (ddd, $1 \mathrm{H}, J=8.3,7.8,1.0 \mathrm{~Hz}, \mathrm{H} 2), 7.78-7.67(\mathrm{~m}, 3 \mathrm{H}), 7.52$ (ddd, $1 \mathrm{H}, J=8.0,7.5,0.8 \mathrm{~Hz}, \mathrm{H} 9), 4.62(\mathrm{q}, J=7.5 \mathrm{~Hz}, 2 \mathrm{H}), 2.76$ $(\mathrm{s}, 3 \mathrm{H}), 1.40(\mathrm{t}, J=7.5 \mathrm{~Hz}, 3 \mathrm{H}) \cdot{ }^{13} \mathrm{C} \times \mathrm{MR}\left(\mathrm{CDCl}_{3}, 62.5\right.$ MHz) $\delta 168.42,161.31,148.34,147.83,145.68,143.90$, $143.37,134.57,130.97,130.29,128.89,128.16,128.04$, $127.77,126.79,126.47,124.22,122.61,61.84,15.55,14.18$. ESI MS $[\mathrm{M}+\mathrm{H}]^{+} 360$. Mass spectrum $\mathrm{m} / \mathrm{z}$ (rel. intensity) $360.1 \mathrm{M}+1,100), 314.3(30), 286.2(30)$. The latter fractions $\left(R_{\mathrm{f}}=0.2\right)$ afforded $10 \mathrm{mg}(14 \%)$ of $7 \mathrm{a}$ as a yellow powder: mp $298^{\circ} \mathrm{C} .{ }^{l} \mathrm{H} \times \mathrm{MR}\left(\mathrm{CDCl}_{3}, 250 \mathrm{MHz}\right) \delta 8.44(\mathrm{~d}$, $1 \mathrm{H}, J=8.3 \mathrm{~Hz}, \mathrm{H1}), 8.38(\mathrm{~d}, 1 \mathrm{H}, J=8.3 \mathrm{~Hz}, \mathrm{H} 4), 8.07(\mathrm{~d}$, $1 \mathrm{H}, J=8.3 \mathrm{~Hz}, \mathrm{H} 7), 8.06(\mathrm{~d}, 1 \mathrm{H}, J=8.3 \mathrm{~Hz}, \mathrm{H} 10), 7.82(\mathrm{td}$, $1 \mathrm{H}, J=8.3,1.0 \mathrm{~Hz}, \mathrm{H} 2), 7.79(\mathrm{td}, 1 \mathrm{H}, J=8.3,1.0 \mathrm{~Hz}, \mathrm{H} 3)$, $7.66(\mathrm{td}, 1 \mathrm{H}, J=8.0,0.8 \mathrm{~Hz}, \mathrm{H} 8), 7.54(\mathrm{t}, 1 \mathrm{H}, J=8.0 \mathrm{~Hz}$, $\mathrm{H} 9), 5.30(\mathrm{~s}, 2 \mathrm{H}), 2.84(\mathrm{~s}, 3 \mathrm{H}) \cdot{ }^{13} \mathrm{C}$ NMR $\left(\mathrm{CDCl}_{3}, 62.5\right.$ $\mathrm{MHz}): \delta 160.56,153.02,150.12,149.18,140.52,134.60$, $131.26,130.31,128.70,128.58,128.28,127.42,126.43$, $123.50,121.22,47.04$ and 15.13. Mass spectrum, $\mathrm{m} / \mathrm{z}$ (rel. intensity) $300.4(\mathrm{M}+1,35), 285(100), 272(80)$. MS (ESI) Cacld. for $\mathrm{C}_{19} \mathrm{H}_{1+1} \mathrm{~N}_{3} \mathrm{O}[\mathrm{M}+\mathrm{H}]^{+} 300$, found 300 . Anal. Caled for $\mathrm{C}_{19} \mathrm{H}_{13} \mathrm{~N}_{3} \mathrm{OH}_{2} \mathrm{O}: \mathrm{C}, 72.367 ; \mathrm{H}, 4.795 ; \mathrm{N}, 12.691$. Found: C. $70.37 ; \mathrm{H}, 4.80 ; \mathrm{N}, 12.69$.

8,9-Difluoro-11-methylluotonin A (7b): Pale yellow needles $(55 \%)$ : mp: $320^{\circ} \mathrm{C} .{ }^{~} \mathrm{H}$ NMR $\left(\mathrm{CDCl}_{3}, 250 \mathrm{MHz}\right) \delta$ $8.43(\mathrm{~d}, 1 \mathrm{H}, J=7.8 \mathrm{~Hz}, \mathrm{Hl}), 8.19\left(\mathrm{dd}, 1 \mathrm{H} . J_{\mathrm{H}-\mathrm{F}}=10.0 \mathrm{~Hz}\right.$, $\left.J_{\text {ord } \mathrm{ru}}=8.0 \mathrm{~Hz}, \mathrm{H} 7\right), 8.10(\mathrm{~d}, 1 \mathrm{H}, J=8.0 \mathrm{~Hz}, \mathrm{H} 4), 7.88-7.80$ $(\mathrm{m}, 2 \mathrm{H}), 7.58(\mathrm{td}, 1 \mathrm{H}, J=7.8,1.3 \mathrm{~Hz}, \mathrm{H} 2), 5.29(\mathrm{~s}, 2 \mathrm{H}), 2.79$ (s, 3H). MS (ESI) Cacld for $\mathrm{C}_{19} \mathrm{H}_{12} \mathrm{~F}_{2} \mathrm{~N}_{3} \mathrm{O}[\mathrm{M}+\mathrm{H}]^{-} 336$. Found 336. Anal. Calcd for $\mathrm{C}_{19} \mathrm{H}_{11} \mathrm{~F}_{2} \mathrm{~N}_{3} \mathrm{O}: \mathrm{C}, 68.06 ; \mathrm{H}, 3.31$; $\mathrm{N}, 11.33$. Found: $\mathrm{C}, 67.97 ; \mathrm{H}, 3.34 ; \mathrm{X}, 11.29$.

9-Bromo-11-methylluotonin A (7e): Pale yellow needles (37\%): mp: $268{ }^{\circ} \mathrm{C} .{ }^{\mathrm{H}} \mathrm{H} \mathrm{NMR}\left(\mathrm{CDCl}_{3}, 250 \mathrm{MHz}\right) \delta 8.41$ (d, $1 \mathrm{H}, J=8.2 \mathrm{~Hz}, \mathrm{H} 1), 8.30(\mathrm{~d}, 1 \mathrm{H}, J=9.1 \mathrm{~Hz}, \mathrm{H} 4), 8.25$, (d, $1 \mathrm{H}, J=1.96 \mathrm{~Hz}, \mathrm{H} 10), 8.08(\mathrm{~d}, 1 \mathrm{H}, J=8.6 \mathrm{~Hz}, \mathrm{H} 7) 7.89-$ $7.80(\mathrm{~m}, 2 \mathrm{H}, \mathrm{H3}$ and $\mathrm{H} 8), 7.57(\mathrm{t}, 1 \mathrm{H}, J=7.3 \mathrm{~Hz}, \mathrm{H} 9), 5.29$ $(\mathrm{s}, 2 \mathrm{H}), 2.79(\mathrm{~s}, 3 \mathrm{H}) .{ }^{13} \mathrm{C} \mathrm{NMR}\left(\mathrm{CDCl}_{3}, 62.5 \mathrm{MHz}\right) ; \delta \mathrm{MS}$ (ESI) Cacld for $\mathrm{C}_{19} \mathrm{H}_{13} \mathrm{Br}_{2} \mathrm{~N}_{3} \mathrm{O}[\mathrm{M}+\mathrm{H}]^{+} 378$. Found 378 . Anal. Calcd for $\mathrm{C}_{19} \mathrm{H}_{12} \mathrm{Br} \mathrm{N}_{3} \mathrm{O}: \mathrm{C}, 60.34 ; \mathrm{H}, 3.20 ; \mathrm{N}, 11.11$. Found: $\mathrm{C}, 60.37 ; \mathrm{H}, 3.24 ; \mathrm{N}, 11.07$.

DNA relaxation assay of Topo $I$. The test compounds 
were dissolved in DMSO at $10 \mathrm{mM}$ as stock solution. The activity of DNA Topo I was determined by assessing the relaxation of supercoiled DNA pBR322. The mixture of 100 ng of plasmid pBR322 DNA and 0.2 units of calf thymus DNA Topo I (Fermentas, USA) was incubated without and with the prepared compounds at $37^{\circ} \mathrm{C}$ for 30 minutes in the relaxation buffer ( $35 \mathrm{mM}$ Tris- $\mathrm{HCl}$ ( $\mathrm{pH} 8.0$ ), $72 \mathrm{mM} \mathrm{KCl}, 5$ $\mathrm{mM} \mathrm{MgCl} 2.5 \mathrm{mM}$ dithiothreitol, $2 \mathrm{mM}$ spermidine, $0.01 \%$ bovine serum albumin). The reaction in the final volume of $10 \mu \mathrm{L}$ was terminated by adding $2.5 \mu \mathrm{L}$ of the stop solution containing $10 \%$ SDS, $0.2 \%$ bromophenol blue, $0.2 \%$ xylene cyanol and $30 \%$ glycerol. DNA samples were then electrophoresed on a $1 \%$ agarose gel at $15 \mathrm{~V}$ for $7 \mathrm{~h}$ with a running buffer of TAE. Gels were stained for $30 \mathrm{~min}$ in an aqueous solution of ethidium bromide $(0.5 \mu \mathrm{g} / \mathrm{mL})$. DNA bands were visualized by transillumination with UV light and were quantitated using Alphalmager ${ }^{\mathrm{TM}}$ (Alpha Innotech Corporation).

DNA relaxation assay of Topo II. The mixture of $100 \mathrm{ng}$ of supercoiled pBR322 plasmid DNA and 0.2 units of human DNA Topo II $\alpha$ (Amersham, USA) was incubated without and with the prepared compounds in the assay buffer ( $10 \mathrm{mM}$ Tris- $\mathrm{HCl}$ ( $\mathrm{pH} 7.9$ ) containing $50 \mathrm{mM} \times \mathrm{aCl}, 5$ $\mathrm{mM} \mathrm{MgCl} \mathrm{l}_{2}, 1 \mathrm{mM}$ EDTA, $1 \mathrm{mM}$ ATP, and $15 \mu \mathrm{g} / \mathrm{mL}$ bovine serum albumin) for $30 \mathrm{~min}$ at $37^{\circ} \mathrm{C}$. The reaction in a final volume of $10 \mu \mathrm{L}$ was terminated by the addition of $3 \mu \mathrm{L}$ of 7 mM EDTA. Reaction products are analyzed on a $1 \%$ agarose gel at $25 \mathrm{~V}$ for $4 \mathrm{~h}$ with a running buffer of TAE. Gels were stained for $30 \mathrm{~min}$ in an aqueous solution of ethidium bromide $(0.5 \mu \mathrm{g} / \mathrm{mL})$. DNA bands were visualized by transillumination with UV light and supercoiled DNA was quantitated using AlphaImager ${ }^{\mathrm{TM}}$ (Alpha Innotech Corporation).

Acknowledgments. Financial support form Korean Research Foundation Grant (KRF-2005-041-E00496) is gratefully acknowledged. DHK and JLL are recipients of BK-21 scholarship.

\section{References}

1. Xiao, P.-G. A Pictorial Enchclopedia of Chinese Medical Herbs (Japanese edition); Chuokoron-sha, Inc.: Tokyo, 1992; Vol III, P 125 .

2. Xiao, P.-G; Qou, G.-L.; Wang, H.-L.; Lui, L.-S.; Zheng, Y.-L.; Jia, Z.-J.; Deng, Z.B. Chinese J. Pharmacol. Toricol 1988, 232.

3. Ma, Z.-Z.; Hano, Y; Nomura, T.; Chen, Y.-J. Heterockdes 1997, 46,541

4. (a) Cagir, A.; Tones, S. H.; Gao, R.; Eisenhauer, B. M.; Hecht, S. M. J. Am. Chem, Soc. 2003, 125, 13628. (b) Ma, Z.-Z.; Hano, Y; Nomura, T.; Chen, Y.-J. Bioorg. Med. Chem. Lett. 2004, I4, 1193.

5. (a) Zhang, Q.; Rivkin, A.; Curran, D. P. J. Am. Chem. Soc. 2002, 124, 5774. (b) Blagg, B. S. J.; Boger, D. L. Tetrahedron 2002, 58 , 6343. (c) Curran, D. P.; Du, W. Org. Leff. 2002, 4, 3215. (d) Comins, D. L.; Nolan, J. M. Org. Leff. 2001, 3, 4255. (e) Toyota, M.; Komori, C.; Ihara, M. J. Org. Chent. 2000, 65, 7110.

6. (a) Twin, H.; Batey, R. A. Org. Lett. 2004, 6, 4913. (b) Chavan, S.
P.; Sivappa, R. Tetrohedron 2004, 60, 9931. (c) Harayama, T; Hori, A.; Serban, G.; Morikami, Y.; Matsumoto, T.; Abe, H.; Takeuchi, Y. Tetrohedron 2004, 60, 10645. (d) Bowman, W. R.; Cloonan, M. O.; Fletcher, A. J.; Stein, T. Org. Bionol. Chem. 2005, 3, 1460. (e) Mhaske, S. B.; Argade, N. P. J. Org. Chem. 2004, 69, 4563-4566. (f) Lee, E. S.; Park, J. G.; Jalung, Y. Tetrahedron Lett. 2003, 44, 1883. (g) Osbone, D.; Stevenson, P. J. Tetwhedron Lett. 2002, 43, 5469. (h) Yadav, I. S.; Reddy, B. V. S. Tew thedron Lem. 2002, 43, 1905. (j) Dallavalle, S.; Merlini, L. Tetrahedron Lett. 2002, 43, 1835. (i) Toyota, M.; Komori, C.; Ihara, M. Heterocveles 2002, 56, 101 . (k) Molina, P.; Tarraga, A.; Gonzalez-Tejero, A. Slnthesis 2000, 11,1523 . (1) Ma, Z.-Z. Hano, Y.; Nomura, T.; Chen, Y.-T. Heteroctcles 1999, 51, 1593. (m) Kelly, T. R.; Chamberland, S.; Silva, R. A. Tetrohedron Lett. 1999, 40, 2723. (n) Wang, H.; Ganesan, A. Tetrahedon Lett. 1998, 39, 9097. (o) Curran, D. P.; Tangirala, R. S. 228th National Meeting of Am. Chem. Soc (August 22-26, Philadelphia, PA) 2004, Abs. ORG 398. (p) Tangirala, R.; Antony, S.; Agama, K.; Pommier, Y.; Curran, D. P. Sinlett 2005, 18, 2843.

7. (a) Ma, Z.; Hano, Y.; Nomura, T.; Chen, Y. Bioorg. Med. Chen. Lett. 2004, 14, 1193. (b) Cagir, A.; Jones, S. H.; Eisenhauser, B. M.; Gao, R.; Hecht, S. M. Bioorg. Med. Chem. Lett. 2004, 14 , 2051. (c) Cagir, A.; Eisenlhauser, B. M.; Gao, R.; Thomas, S. J.; Hecht, S. M. Bioorg. Med. Chem. 2004, 12,6287. (d) Dallavalle, S.; Merlini, L.; Beretta, G. L.; Tinelli, S.; Zunino, F. Bioorg. Med. Chem. Lett. 2004, 14, 5757. (e) Cheng, K.; Rahier, $N$; Eisenhauser, B. M.; Gao, R.; Thomas, S. J.; Hecht, S. M. J. Am. Chem. Soc. 2005, 127, 838. (f) For a review see: Ma, Z.-Z.; Hano, Y; Nomura, T. Heterocycles 2005, 65, 2203.

8. Chang, H. W. Kim, S. I.; Jung, H. J.; Jalung, Y. Heterocycles 2003, 60,1359

9. (a) Staker, B. L.; Hjerrild, K.; Feese, M. D.; Behnke, C. A.; Burgin, Ir., A. B.; Stewart, K. Proc. Nonl. Acall Sci. U.S.A. 2002, 99, 15387. (b) Staker, B. L.; Feese, M. D.; Cushman, M.; Pommier, Y.; Zembower, D.; Stewart, L.; Burgin, A. B. J. Mfed. Chem. 2005, 48, 2336. (c) Xiao, X.; Antony, S.; Pommier, Y.; Cushunan, M. J. Med. Chen. 2006, 49, 1408. (d) Redinbo, M. R,; Stewart, L.; Kuhn, P.; Champoux, J. J.; Hol, W. G. Science 1998, 279, 1504. (e) Stewart, L.; Redinbo, M. R.; Qu, X.; Hol, W. G;; Champoux, J. J. Science 1998, 279, 1534.

10. (a) Jung, H. W.; Oh, J. S.; Lee, S. H.; Liang, J. L.; Kim, D. H.; Rahman, A. F. M. M.; Jalung, Y. Bull. Korean Chem. Soc. 2007, 28, 1863. (b) Zhao, L.-X.; Park, H.-g.; Jew, S.-s.; Lee, M. K.; Kim, Y. J; Thapa, P; Karki, R.; Jahung, Y.; Jeong, B.-S.; Lee, E.-S. Bull. Korem Chem. Soc. 2007, 28,970.

11. (a) Friedländer, P. Ber. 1882, 15, 2572. (b) For a review see: Cheng, C.-C.; Yang, S.-J. Org. React. 1982, 28,37 and Thummel, R. P. Symlet 1992, 1 , and references therein.

12. (a) Opie, I. W.; Smith, L. I. Org. Sin. Col. Vol III 1955, 56. (b) Majewicz, G.; Caluwe, O. A.J. Org. Chen. 1974, 39, 720.

13. (a) Shaw, K. N. F.; Gudmundson, A.; Morris, A. G. Biochen. Prep. 1962, 9, 12. (b) Katritzky, A. R.; Wang, Z.; Hall, C. D.; Ji, Y.; Aklumedov, N. G. Tetrahedron Lett. 2002, 43, 3449. (c) Mosettig, E.; Czadek, K. Monat. Chem. 1931, 57, 291. (d) Shen, W; Coburn, C. A.; Bornunan, W. G; Danishefsky, S. I. J. Org. Chem. 1993, 58,611.

14. Fukuda, M.; Nishio, K.; Kanazawa, F.; Ogasawara, H.; Ishida, T.; Arioka, H.; Bojanowșii, K.; Oka, M.; Saijo, N. Cancer Res. 1996, 56,789 .

15. (a) Skehan, P.; Streng, R.; Scudiero, D.; Monks, A.; McMahon, J.; Vistica, D.; Warrenm, I. T.; Bokesch, H.; Kenney, S.; Boyd, M. R. J. Natl. Concer Inst. 1990, 82, 1107. (b) Kang, D. H.; Kim, J. S.; Jung, M. J.; Lee, E. S.; Jahng, Y; Kwon, Y,; Na, Y. Bioong. Hed. Chem. Letr. 2008, 18,1520 . 